\title{
Temperature measurement by means of fiber specklegram sensors (FSS)
}

\section{Medición de temperatura mediante specklegramas de fibra ópica (FSS)}

\author{
Luis F. Castaño ${ }^{1}$, Luis C. Gutierrez ${ }^{1}$, Jairo C. Quijanoํㅜ , Jorge A. Herrera-Ramírez ${ }^{1,2}$, \\ Alejandro Hoyos ${ }^{1}$, Francisco J. Vélez ${ }^{3}$, Víctor H. Aristizabal ${ }^{3}$, Luiz C. Da Silva-Nunez ${ }^{4}$, \\ Jorge A. Gómez ${ }^{1^{*}}$ \\ 1. Grupo de investigación de Física Básica y Aplicada, Politécnico Colombiano Jaime Isaza Cadavid, Medellín, \\ Colombia \\ 2. Facultad de Ingenierías, Instituto Tecnológico Metropolitano, Medellín, Colombia \\ 3. Grupo de Investigación TERMOMEC, Universidad Cooperativa de Colombia, Medellín, Colombia \\ 4. Departamento de Ingeniería Mecánica, Universidad Federal Fluminense, São Paulo, Brasil \\ ${ }^{*}$ E) E-mail: jagomez@elpoli.edu.co
}

Received: 18/12/2017 Accepted: 06/08/2018

DOI: $10.7149 /$ OPA.51.3.50306

\begin{abstract}
:
In this work, a technique for temperature measurement using non-holographic fiber optic specklegrams is demonstrated experimentally. In the experimental scheme, a $632 \mathrm{~nm}$ laser source is coupled to a mono-multi-mono mode fiber arrangement, which produces a filtering effect that is used as an optical transduction mechanism. Thermal perturbations between $25^{\circ} \mathrm{C}$ and $60^{\circ} \mathrm{C}$ were applied to the fiber array, finding a linear response of the system and a sensitivity superior to the previously reported for studies in similar systems. Due to the low cost and simplicity of the technique, these results are of great interest for the implementation of this type of optical fiber sensors in a large number of engineering applications.
\end{abstract}

Key words: Fiber Specklegram Sensors, Thermal Perturbations, Optical Fiber sensors.

\section{RESUMEN:}

En este trabajo, una técnica para la medición de temperatura mediante specklegramas de fibra óptica no-holográficos es demostrada experimentalmente. En el esquema experimental, una fuente láser de $632 \mathrm{~nm}$ es acoplada a un arreglo de fibras mono-multi-mono modo, lo cual produce un efecto de filtrado que es usado como mecanismo de transducción óptica. Perturbaciones térmicas entre los $25^{\circ} \mathrm{C}$ y los $60^{\circ} \mathrm{C}$ fueron aplicadas al arreglo de fibras, encontrando una respuesta lineal del sistema y una sensibilidad superior a las reportadas anteriormente para estudios basados en sistemas similares. Debido al bajo costo y simplicidad de la técnica, estos resultados son de gran interés para la implementación de este tipo de sensores de fibra óptica en una gran cantidad de aplicaciones de ingeniería.

Palabras clave: Specklegramas de Fibra Óptica, Perturbaciones Térmicas, Sensores de Fibra Óptica.

\section{REFERENCES AND LINKS / REFERENCIAS Y ENLACES}

[1] K. J. Gasvik, Optical Metrology, 3rd ed. Chichester, England: John Wiley \& Sons Ltd, (2002).

[2] Y. Y. Hung, "Displacement and strain measurement," in Speckle metrology, R. K. Erf, Ed. New York: Academic Press, Inc., pp. 51-71 (1978).

[3] R. Jones and C. Wykes, Holographic and Speckle Interferometry. Cambridge University Press, (1989). 
[4] B. E. a Saleh and M. C. Teich, Fundamentals of Photonics, vol. 5. New York, USA: John Wiley \& Sons, Inc., (1991).

[5] F. T. S. Yu and S. Yin, Fiber Optic Sensors. New York: Marcel Dekker, Inc., (2002).

[6] B. Wang, C. Huang, R. Guo, and F. T. S. Yu, "A novel fiber chemical sensor using inner-product multimode fiber speckle fields," in Proceedings of SPIE - The International Society for Optical Engineering, p. 299 (2003).

[7] Y. Liu and L. Wei, "Low-cost high-sensitivity strain and temperature sensing using graded-index multimode fibers," Appl. Opt., 46, pp. 2516-2519 (2007).

[8] I. P. Johnson, D. J. Webb, K. Kalli, M. C. J. Large, and A. Argyros, "Multiplexed FBG sensor recorded in multimode microstructured polymer optical fibre,", 7714, p. 77140D (2010).

[9] D. Monzon-Hernandez, V. P. Minkovich, and J. Villatoro, "High-temperature sensing with tapers made of microstructured optical fiber," IEEE Photonics Technol. Lett., 18, pp. 511-513, (2006).

[10] Y. Peng, J. Hou, Z. Huang, and Q. Lu, "Temperature sensor based on surface plasmon resonance within selectively coated photonic crystal fiber," Appl. Opt., 51, p. 6361, (2012).

[11] S. Wu, S. Yin, and F. T. S. Yu, “Sensing with fiber specklegrams," Appl. Opt., 30, p. 4468, (1991).

[12] F. T. S. Yu, J. Zhang, S. Yin, and P. B. Ruffin, "Analysis of a fiber specklegram sensor by using coupledmode theory," Appl. Opt., 34, p. 3018, (1995).

[13] F. T. S. Yu, S. Yin, J. Zhang, and R. Guo, “Application of a fiber-speckle hologram to fiber sensing," Appl. Opt., vol. 33, p. 5202, (1994).

[14] J.A. Gomez, H. Lorduy G., and Á. Salazar, "Improvement of the dynamic range of a fiber specklegram sensor based on volume speckle recording in photorefractive materials," Opt. Lasers Eng., vol. 49, no. 3, pp. 473-480 (2011).

[15] J. A. Gómez, H. Lorduy G., and Á. Salazar, "Influence of the volume speckle on fiber specklegram sensors based on four-wave mixing in photorefractive materials," Opt. Commun., 284, pp. 10081014, (2011).

[16] J. A. Gómez and Á. Salazar, "Self-correlation fiber specklegram sensor using volume characteristics of speckle patterns," Opt. Lasers Eng., 50, pp. 812-815, (2012)

[17] A. Malki, R. Gafsi, L. Michel, M. Labarrère, and P. Lecoy, "Impact and vibration detection in composite materials by using intermodal interference in multimode optical fibers," Appl. Opt., 35, p. 5198, (1996).

[18] B. Wang, R. Guo, S. Yin, and F. T. S. Yu, “Chemical Sensing with Hetero-Core Fiber Specklegram," J. Hologr. Speckle, 1, pp. 53-57, (2004).

[19] F. T. S. Yu, M. Wen, S. Yin, and C.-M. Uang, "Submicrometer displacement sensing using inner-product multimode fiber speckle fields," Appl. Opt., 32, p. 4685, (1993).

[20] E. Fujiwara, Y. T. Wu, and C. K. Suzuki, "Vibration-based specklegram fiber sensor for measurement of properties of liquids," Opt. Lasers Eng., 50, pp. 1726-1730, (2012).

[21] J. Li, H. Cai, J. Geng, R. Qu, and Z. Fang, "Specklegram in a multiple-mode fiber and its dependence on longitudinal modes of the laser source," Appl. Opt., 46, p. 3572, (2007).

[22] Y. Wang, H. Cai, R. Qu, Z. Fang, E. Marin, and J.-P. Meunier, "Specklegram in a grapefruit fiber and its response to external mechanical disturbance in a single-multiple-single mode fiber structure," Appl. Opt., 47, p. 3543, (2008).

[23] A. Kumar, R. K. Varshney, S. Antony C, and P. Sharma, "Transmission characteristics of SMS fiber optic sensor structures," Opt. Commun., 219, pp. 215-219, (2003).

[24] E. Fujiwara, Y. T. Wu, M. F. M. dos Santos, E. A. Schenkel, and C. K. Suzuki, "Development of a tactile sensor based on optical fiber specklegram analysis and sensor data fusion technique," Sensors Actuators A Phys., 263, pp. 677-686, (2017).

[25] L. Rodriguez-Cobo, M. Lomer, and J.-M. Lopez-Higuera, “Fiber Specklegram-Multiplexed Sensor," J. Light. Technol., 33, , pp. 2591-2597, (2015).

[26] E. Fujiwara, M. F. Marques dos Santos, and C. K. Suzuki, "Optical fiber specklegram sensor analysis by speckle pattern division,” Appl. Opt., 56, no. 6, p. 1585, (2017).

[27] N. Darío Gómez and J. A. Gómez, "Effects of the speckle size on non-holographic fiber specklegram sensors," Opt. Lasers Eng., 51, pp. 1291-1295, (2013). 
[28] V. H. Aristizabal, A. Hoyos, E. Rueda, N. D. Gomez, and J. A. Gomez, "Effect of wavelength on metrological characteristics of non-holographic fiber specklegram sensor," Photonic Sensors, 5, (2015).

[29] V. H. Arístizabal, F. J. Vélez, E. Rueda, N. D. Gómez, and J. A. Gómez, "Numerical modeling of fiber specklegram sensors by using finite element method (FEM)," Opt. Express, 24, pp. 27225-27238, (2016).

[30] Z. Zhang and F. Ansari, "Fiber-optic laser speckle-intensity crack sensor for embedment in concrete," Sensors Actuators A Phys., 126, , pp. 107-111, (2006).

[31] L. Rodriguez-Cobo, M. Lomer, and J. M. Lopez-Higuera, "Fiber specklegram sensors sensitivities at high temperatures," in Proceedings of SPIE - The International Society for Optical Engineering, p. 96347, (2015).

[32] G. T. Mase and G. E. Mase, Continuum for Engineers, 2 Ed. Boca Raton: CRC Press, (1999).

[33] M. Bass, E. W. Van-Stryland, D. R. Williams, and W. L. Wolfe, Handbook of Optics, Vol. II: Devices, Measurements and Properties, 2nd ed. The United States of America: McGraw-Hill, Inc., (1995).

[34] V. H. Aristizabal, F. J. Velez, and P. Torres, "Numerical model and analysis of optical fibers with internal electrodes," Rev. Colomb. Física, 38, , pp. 173-176, (2006).

[35] K. Masuda, A. Tate, M. Ishida, T. Suzuki, and H. Tsuda, "Beam steering type of $1: 4$ optical switch using thermo-optic effect," Opt. Rev., 13, pp. 184-188, (2006).

\section{Introducción}

Cuando la radiación laser es insertada en una fibra óptica multimodo, los efectos de propagación producen a la salida de la fibra un patrón de speckle (moteado), el cual contiene información sobre el estado espacial de la fibra óptica y que puede ser usado en aplicaciones metrológicas [1-5]. De esta manera, pequeñas perturbaciones sobre la fibra multi-modo producirán cambios en la distribución espacial del patrón de speckle que pueden ser detectados mediante técnicas de correlación o mediante cambios locales de intensidad [6, 7]. Los sistemas de sensado basados en patrones de speckle producidos en fibras ópticas multimodo son conocidos como Sensores de Specklegramas de Fibra Óptica (FSS por sus siglas en inglés). En contraste, otros desarrollos se han venido alcanzando en la detección de cambios térmicos, aunque en muchas situaciones estos demandan el uso de fibras especiales como es el caso de las fibras microestructuradas [8-10].

Los sensores FSS han sido ampliamente estudiados mediante arreglos holográficos cuyo medio de registro es un material foto refractivo [11-16], sin embargo, más recientemente, la importancia de este tipo de sistemas en esquemas no-holográficos ha sido explorada en la medición de múltiples parámetros de interés en ingeniería [17-26]. Alrededor de los arreglos no-holográficos, dos posibilidades han sido reportadas. En el primer caso, herramientas de procesamiento de imágenes son usadas para calcular algoritmos de correlación entre los patrones de speckle perturbados que son capturados mediante cámaras CCD a la salida de la fibra multimodo [17-19]. En el segundo caso, esquemas experimentales de fibras mono-multi-mono modo, han sido usados para generar y filtrar el patrón de speckle que se genera en la fibra multimodal, lo que permite configurar esquemas de FSS interrogados por variación de potencia óptica. En este sentido, nuestro grupo demostró experimentalmente que el tamaño promedio del grano de speckle generado en la fibra multimodo, tiene un fuerte efecto sobre las características metrológicas de un sensor de perturbaciones mecánicas [27, 28]. Así mismo, desarrollamos un modelo numérico que permite corroborar este hallazgo experimental y que facilita el diseño de este tipo de sensores [29].

Los FSS interrogados mediante la variación de potencia óptica, tienen muchas ventajas sobre los FSS holográficos y no-holográficos basados en procesamiento de imágenes [23-28]. Dentro de sus principales ventajas se destacan: la posibilidad de tener sistemas muy compactos y totalmente a fibra, tiempo de respuesta muy bajo, estabilidad y bajo costo [30]. En este trabajo exploramos experimentalmente la medición de perturbaciones térmicas alrededor de la temperatura ambiente. Nuestro esquema experimental, nos permitió realizar medidas entre los $25^{\circ} \mathrm{C}$ y los $60^{\circ} \mathrm{C}$ con una resolución de $5^{\circ} \mathrm{C}$, lo que muestra un desempeño muy superior a resultados reportados anteriormente para sistemas similares, en los cuales se demostró la utilidad de la técnica en ambientes con temperaturas mucho más altas, resoluciones de alrededor de los $50^{\circ} \mathrm{C}$ [31] y con requerimientos adicionales de adquisición y análisis de imágenes. Adicionalmente, se encontró un comportamiento con alta linealidad en el rango de medida, lo 
que facilita la implementación de los resultados en problemas de ingeniería y abre las posibilidades para profundizar en estudios que permitan mejorar las características metrológicas del sistema.

\section{Análisis teórico}

En aproximación de campo cercano, la distribución espacial de intensidad del campo a la salida de una fibra óptica multimodo se puede escribir en coordenadas polares en el punto $(r, \theta)$ como [27]

$$
\begin{aligned}
I=\frac{1}{2} Y \sum_{m=1}^{M} A_{m}^{2} B_{n m}^{2}\left(U_{m} R\right) \cos ^{2}\left(n_{m} \theta\right) \\
\quad+\sum_{m=1}^{M} \sum_{l=m+1}^{M} A_{m} A_{l} B_{n m}\left(U_{m} R\right) B_{n l}\left(U_{l} R\right) \cos \left(n_{m} \theta\right) \cos \left(n_{l} \theta\right) \times \cos \left(\Delta \beta_{m l} z-\Delta \phi_{m l}\right),
\end{aligned}
$$

donde $\Delta \beta_{m l}=\beta_{m}-\beta_{l}$ es la diferencia de las constantes de propagación, $\Delta \phi_{m l}=\phi_{m}-\phi_{l}$ es la diferencia de las constantes de fase, $A_{m}$ y $A_{l}$ son las amplitudes de los modos $m$ y $l$, respectivamente. $R=a / r$ es el radio normalizado siendo $a$ el radio del núcleo de la fibra óptica, $Y=n \sqrt{\varepsilon_{0} / \mu_{0}}$ es la admitancia característica del núcleo, donde $n$ es el índice de refracción del núcleo, $U_{m}=a \sqrt{k^{2} n^{2}-\beta_{m}^{2}}$ es el parámetro modal, $k=2 \pi / \lambda_{0}$ es el número de onda de la luz en el vacío y $B_{n m}\left(U_{m} R\right)$ es la función de Bessel de primera especie.

Cuando la fibra es perturbada, se asume que los cambios en el patrón de speckle se deben principalmente a cambios de fase y que la intensidad total del patrón de speckle en la fibra es constante. Ahora, considerando que al sensor de specklegramas a fibra o FSS (Fiber Specklegram Sensor) se le aplica una perturbación en una pequeña región de longitud $L$, los cambios de fase entre el modo $m$ y $l$ se pueden escribir como $\delta\left(\Delta \beta_{m l} L\right)$. De esta manera, si las variaciones de fase son pequeñas, la nueva distribución de intensidad del patrón a la salida de la fibra multimodo en el punto $(r, \theta)$ es:

$$
\begin{aligned}
I^{\prime}=\frac{1}{2} Y \sum_{m=1}^{M} A_{m}^{2} B_{n m}^{2}\left(U_{m} R\right) \cos ^{2}\left(n_{m} \theta\right)+ \\
\quad+\sum_{m=1}^{M} \sum_{l=m+1}^{M} A_{m} A_{l} B_{n m}\left(U_{m} R\right) B_{n l}\left(U_{l} R\right) \cos \left(n_{m} \theta\right) \cos \left(n_{l} \theta\right) \times \cos \left(\Delta \beta_{m l} z-\Delta \phi_{m l}\right) \\
\pm \delta\left(\Delta \beta_{m l} L\right) \operatorname{sen}\left(\Delta \beta_{m l} z-\Delta \phi_{m l}\right) .
\end{aligned}
$$

Entonces, para calcular los cambios en el patrón de intensidad $\Delta I$ se usan las ecuaciones (1) y (2):

$$
\begin{aligned}
\Delta I=I-I^{\prime}= & \sum_{m=1}^{M} \sum_{l=m+1}^{M} A_{m} A_{l} B_{n m}\left(U_{m} R\right) B_{n l}\left(U_{l} R\right) \cos \left(n_{m} \theta\right) \cos \left(n_{l} \theta\right) \times \delta\left(\Delta \beta_{m l} L\right) \\
& \times \operatorname{sen}\left(\Delta \beta_{m l} z-\Delta \phi_{m l}\right) .
\end{aligned}
$$

De (3) se puede concluir que las variaciones en el patrón de intensidad son debidas a las diferencias de fase de los modos $m$ y $l$. Entonces, los cambios de fase se pueden escribir como $\Delta \beta_{m l}=n D_{m l}$, donde $D_{m l}$ es una constante proporcional asociada con el cambio de fase entre los modos $m$ y $l$. De esta forma, los cambio en la constante de propagación en la dirección $i(i=x, y o z)$ debido a una perturbación en un segmento $L$ sobre la fibra se puede expresar como

$$
\delta_{i}\left(\Delta \beta_{m l} L\right)=D_{m l} \delta_{i}(n L) .
$$


En la ecuación (4) se puede observar que los cambios en las constantes de propagación de los modos que viajan a través de la fibra se deben a cambios en la longitud de camino óptico $\delta_{i}(n L)$. Es posible demostrar que cuando una perturbación es aplicada al segmento de fibra multimodo $\delta_{i}(n L)$ está dado por

$$
\delta_{i}(n L)=\delta_{i} n L+n \delta_{i} L=L\left(\Delta n_{i}+n S_{i}\right)
$$

donde, $\Delta n_{i}=n_{i}-n_{0} \approx-\frac{n_{0}^{3}}{2} P_{i k} S_{k}=-C_{i k} \sigma_{k}$ (aquí se indica en notación tensorial) es el cambio del índice de refracción siendo el material isotrópico en un momento inicial con índice $n_{0}, S_{i}$ es la deformación mecánica (mechanical strain en inglés) y $\sigma_{i}$ es la tensión o esfuerzo mecánico (mechanical stress en inglés) en la dirección principal $i . P_{i k}$ es el tensor de deformación mecánica y $C_{i k}$ es el tensor de esfuerzo mecánico [29]. En forma más simplificada:

$$
\begin{aligned}
& \delta_{x}(n L)=L\left(\Delta n_{x}+n S_{x}\right) \\
& \delta_{y}(n L)=L\left(\Delta n_{y}+n S_{y}\right) \\
& \delta_{z}(n L)=L\left(\Delta n_{z}+n S_{z}\right)
\end{aligned}
$$

donde bajo la suposición de deformación plana (plane strain approximation en inglés), es decir $S_{z}=0$ se tiene que los cambios de índice de refracción debido a efectos mecánicos son [29, 32]:

$$
\begin{gathered}
\Delta n_{x}^{\text {mec }}=-\frac{n_{0}^{3}}{2}\left(P_{11} S_{x}+P_{12} S_{y}\right) \\
\Delta n_{y}^{\text {mec }}=-\frac{n_{0}^{3}}{2}\left(P_{12} S_{x}+P_{11} S_{y}\right) \\
\Delta n_{z}^{\text {mec }}=-\frac{n_{0}^{3}}{2} P_{12}\left(S_{x}+S_{y}\right),
\end{gathered}
$$

donde $P_{11}$ y $P_{12}$ corresponden a los coeficientes elasto-ópticos, cuyos valores para la sílice son $0.121 \mathrm{y}$ 0.270 respectivamente. Ahora, si hacemos uso de la termo-elasticidad lineal [32,33], para considerar una perturbación termo-mecánica, es decir, cambios del índice debido a la dilatación del material por el cambio de temperatura, entonces $S_{i}=\alpha\left(T-T_{0}\right)$ con $i=x, y$, donde $\alpha=\frac{1}{L} \frac{d L}{d T}$ es el coeficiente lineal de expansión térmica del material y $T_{0}$ es la temperatura del material en un momento inicial. Quedando las expresiones en (7) como:

$$
\begin{gathered}
\Delta n_{x}^{T M}=-\frac{n_{0}^{3}}{2} \alpha\left(P_{11}+P_{12}\right)\left(T-T_{0}\right) \\
\Delta n_{y}^{T M}=-\frac{n_{0}^{3}}{2} \alpha\left(P_{12}+P_{11}\right)\left(T-T_{0}\right) \\
\Delta n_{z}^{T M}=-\frac{n_{0}^{3}}{2} 2 \alpha P_{12}\left(T-T_{0}\right) .
\end{gathered}
$$

Además, considerando los cambios del índice debido al cambio de temperatura, es decir, una perturbación termo-óptica $\Delta n_{i}^{T O}=\mathrm{C}_{T O}\left(T-T_{0}\right)$ donde $\mathrm{C}_{T O}$ es el coeficiente termo-óptico (Thermo-Optic Coefficient) y se define como $d n / d T$. Finalmente, teniendo en cuenta las dos contribuciones anteriores se obtiene:

$$
\begin{gathered}
\Delta n_{x}=n_{x}-n_{0}=\left[\mathrm{C}_{T O}-\frac{n_{0}^{3}}{2} \alpha\left(P_{11}+P_{12}\right)\right]\left(T-T_{0}\right) \\
\Delta n_{y}=n_{y}-n_{0}=\left[\mathrm{C}_{T O}-\frac{n_{0}^{3}}{2} \alpha\left(P_{12}+P_{11}\right)\right]\left(T-T_{0}\right) \\
\Delta n_{z}=n_{z}-n_{0}=\left[\mathrm{C}_{T O}-\frac{n_{0}^{3}}{2} 2 \alpha P_{12}\right]\left(T-T_{0}\right) .
\end{gathered}
$$

En las ecuaciones (9), si los cambios de temperatura son pequeños, se puede despreciar el término que contiene los coeficientes elasto-ópticos, debido a que estos son mucho menores que uno [29, 34], 
obteniendo la ecuación simplificada en (10), la cual nos muestra un comportamiento aproximadamente isotrópico del cambio del índice por efectos térmicos [35].

$$
\Delta n \approx \Delta n_{x}=\Delta n_{y}=\Delta n_{z} \approx \mathrm{C}_{T O}\left(T-T_{0}\right)
$$

Entonces, finalmente las ecuaciones en (6) quedan

$$
\delta(n L)=\delta_{x}(n L)=\delta_{y}(n L)=\delta_{z}(n L) \approx L\left(\mathrm{C}_{T O}+n_{0} \alpha\right)\left(T-T_{0}\right) .
$$

Al reemplazar la ecuación (11) en (3), se puede observar que los cambios de la intensidad en el patrón de speckle varían linealmente con el cambio de temperatura de la fibra óptica. En conclusión, cuando la fibra óptica es perturbada, el patrón de speckle a la salida de la fibra es ligeramente modificado por los cambios de fase en la fibra óptica multimodo. Estas modificaciones producen variaciones locales de intensidad lo que genera pequeñas deformaciones en el patrón de speckle de la fibra. Algo similar se ilustra en la Figura 1, correspondiente a un trabajo que hemos publicado previamente [29], donde la intensidad es perturbada localmente deformando ligeramente el patrón de speckle de la fibra para el caso de una perturbación mecánica. Más aún, si se usa una fibra de filtrado (fibra monomodo) con un tamaño de núcleo apropiado al final de la fibra multimodo, es posible traducir apropiadamente un cambio local en el patrón de speckle en un cambio en la potencia óptica al final de dicha fibra monomodo que es la que lleva la luz al fotodetector. De esta manera, es posible medir las perturbaciones térmicas que alteran el estado de la fibra multimodo.
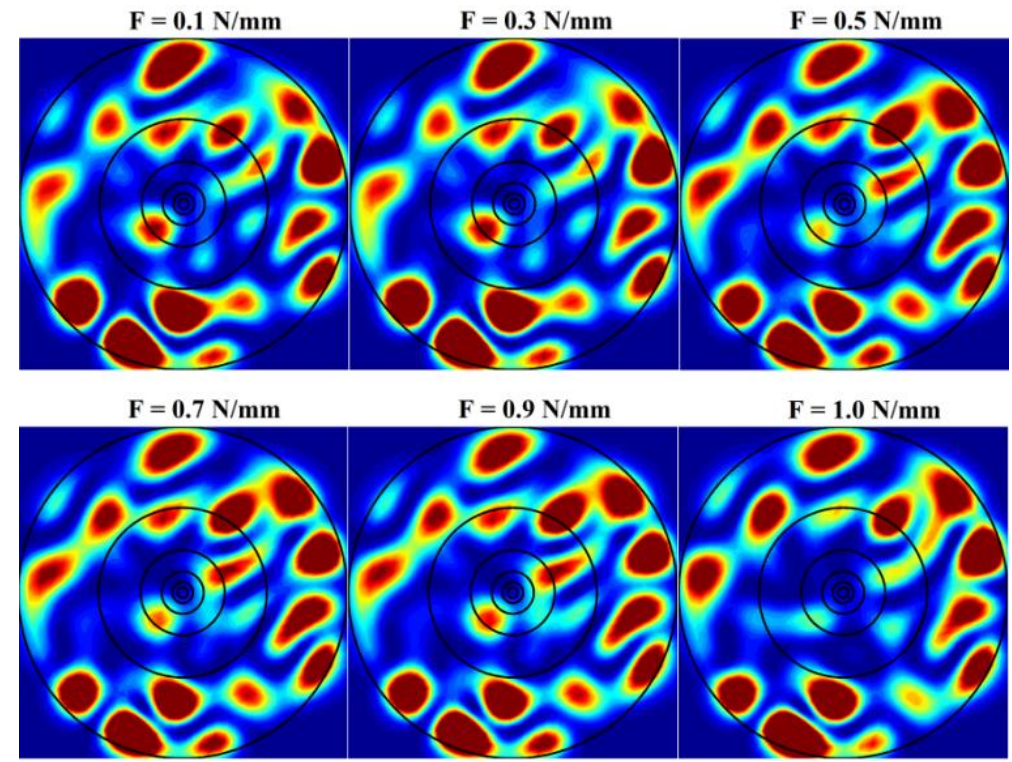

Fig. 1. Imágenes de patrones de speckle en una fibra multimodo simulados numéricamente a $1630 \mathrm{~nm}$ para varios valores de fuerza aplicada. Los círculos negros indican los posibles tamaños de núcleo para la fibra monomodo de filtrado. Tomado de resultado publicado en [29].

\section{Análisis experimental y resultados}

El sistema de medida implementado se conformó mediante un arreglo de fibras mono-multi-mono modo, que fueron conectados a un láser de $632 \mathrm{~nm}$ y a un fotodetector THORLABS DET01CFC/M. En el esquema experimental se usó una fibra multimodo estándar $(62.5 \mu \mathrm{m} / 125 \mu \mathrm{m})$ como fibra transductora y una fibra monomodo estándar $(8 \mu \mathrm{m} / 125 \mu \mathrm{m})$ como fibra de iluminación y filtrado. Para perturbar de manera controlada el arreglo de fibras, se desarrolló un control de temperatura basado en la plataforma ARDUINO ${ }^{\circ}$, lo que permitió elevar de manera controlada la temperatura hasta $\operatorname{los} 60^{\circ} \mathrm{C}$, permitiendo la estabilización térmica del ambiente y su homogeneidad, según se constató con un termopar como dispositivo de medida de referencia. En la Figura 2 se presenta un esquema y una foto del prototipo experimental. 

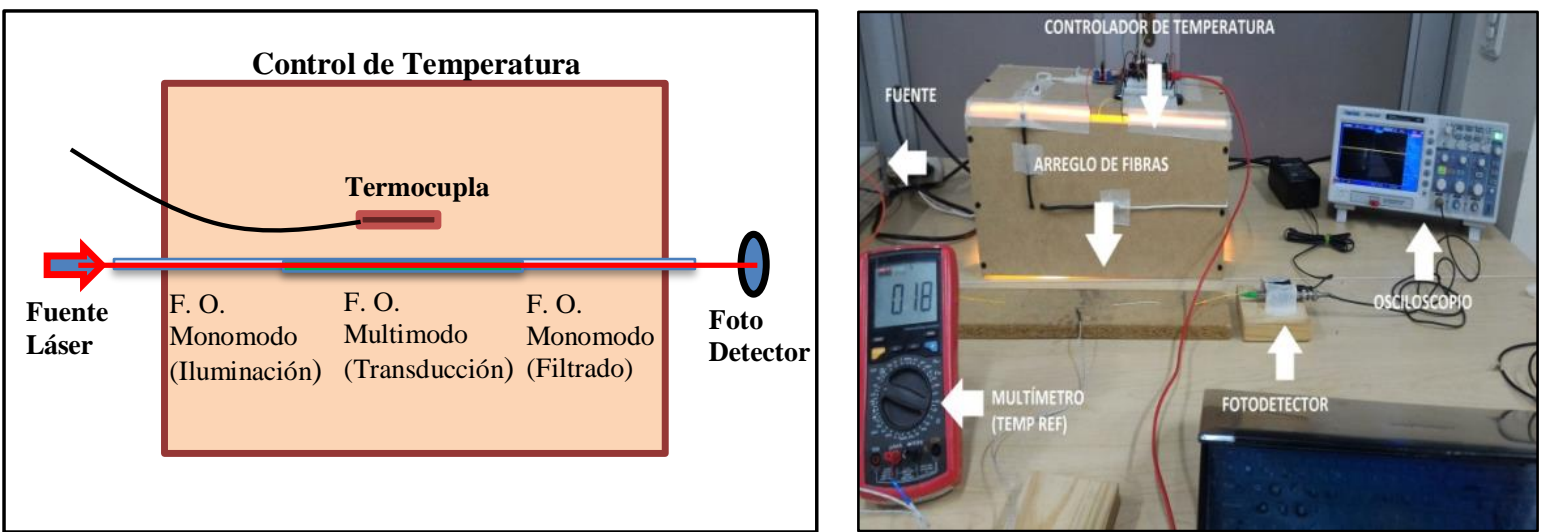

Fig. 2. Arreglo experimental del FSS para medición de temperatura.

En la Figura 3 se muestra la respuesta del sistema de medición junto a la curva de ajuste hallada mediante regresión lineal. Allí se puede observar que el sistema exhibe un comportamiento altamente lineal en el rango estudiado con incrementos en la temperatura de $5^{\circ} \mathrm{C}$, coincidiendo con la teoría expuesta en la sección 2, donde se muestra que para cambios de temperatura no muy grandes las variaciones de intensidad se dan linealmente.

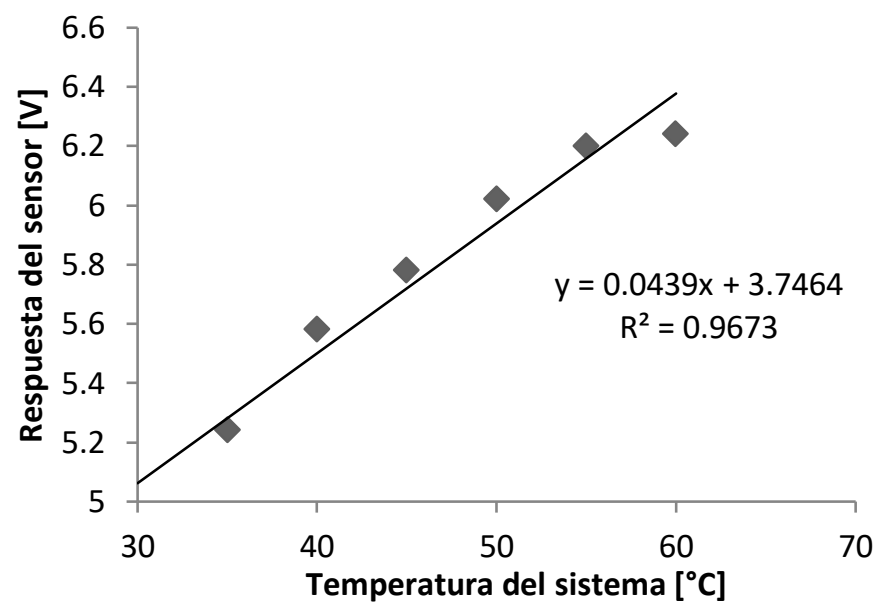

Fig. 3. Respuesta del sistema de medición para cambios de temperatura

\section{Conclusiones}

En este trabajo, se usó la técnica FSS para la medición de temperatura. Con el esquema experimental desarrollado fue posible realizar medidas desde temperatura ambiente hasta $\operatorname{los} 60^{\circ} \mathrm{C}$ con una resolución de $5^{0} \mathrm{C}$. Estos resultados resultan ser prometedores, ya que en estudios realizados con esquemas similares se ha reportado la posibilidad de obtener medidas para temperaturas mucho más elevadas, entre los $100^{\circ} \mathrm{C}$ y los $800^{\circ} \mathrm{C}$ y con resoluciones de alrededor de $50^{\circ} \mathrm{C}$, como se evidencia en [31], y que a diferencia de estos sistemas no requiere adquisición y análisis de imágenes sino una lectura directa de potencia óptica. Por tal razón, este trabajo abre la posibilidad de consolidar sistemas de medida basados en fibra óptica de muy bajo costo y muestra la importancia de continuar estudiando este fenómeno para alcanzar mejores sensibilidades.

\section{Agradecimientos}

Los autores agradecen al Politécnico Colombiano Jaime Isaza Cadavid, a la Universidad Cooperativa de Colombia y a la Universidade Federal Fluminense. V.H. Aristizábal y F.J. Vélez agradecen el apoyo financiero de la Universidad Cooperativa de Colombia mediante el proyecto con código INV1898. 